Trans

continentales
Transcontinentales

Sociétés, idéologies, système mondial

$10 / 11 \mid 2011$

La ruée vers la terre

\title{
Riad al-Solh en son temps (Riyâd Al-Sulh fî zamâni- hi), Beyrouth, Dar An-Nahar, 2011
}

Conclusion de l'ouvrage

\section{Ahmad Beydoun}

Traducteur : Michel Tabet

\section{(2) OpenEdition}

\section{Journals}

Édition électronique

URL : http://journals.openedition.org/transcontinentales/1143

DOI : 10.4000/transcontinentales. 1143

ISBN : : 978-2-7351-1572-3

ISSN : $1775-397 X$

\section{Éditeur}

Editions de la maison des sciences de l'homme

\section{Référence électronique}

Ahmad Beydoun, «Riad al-Solh en son temps (Riyâd Al-Sulh fî zamâni-hi), Beyrouth, Dar An-Nahar, $2011 »$, Transcontinentales [En ligne], 10/11 | 2011, mis en ligne le 19 octobre 2011, consulté le 08 septembre 2020. URL : http://journals.openedition.org/transcontinentales/1143 ; DOI : https://doi.org/ 10.4000/transcontinentales. 1143

Ce document a été généré automatiquement le 8 septembre 2020

Tous droits réservés 


\section{Riad al-Solh en son temps (Riyâd Al- Sulh fî zamâni-hi), Beyrouth, Dar An-Nahar, 2011}

Conclusion de l'ouvrage

\section{Ahmad Beydoun}

Traduction : Michel Tabet

\section{NOTE DE L'ÉDITEUR}

Ahmad Beydoun est né en 1943 à Bint Jbeil au Sud-Liban. Après un doctorat soutenu à l'université de la Sorbonne en 1982, il a enseigné la sociologie culturelle à l'Université libanaise jusqu'en 2007. Il est l'auteur d'une œuvre remarquable, écrite en arabe et en français, qui traite de sujets variés, l'historiographie et le système politique libanais, l'immigration chiite aux Etats-Unis, la guerre civile au Liban ou les transformations de la culture et de la langue arabes. Parmi ses ouvrages en français, citons Identité confessionnelle et temps social chez les historiens libanais (Beyrouth, Publications de l'Université libanaise, 1984), Le Liban. Itinéraires dans une guerre incivile (Paris/Beyrouth, Karthala/Cermoc, 1993), La dégénérescence du Liban ou la Réforme orpheline (Arles, Actes Sud/Sindbad, 2009).

Son dernier livre, Riyâd al-Sulh fî zamâni-hi [Riad al-Solh en son temps] (Beyrouth, Dar An-Nahar, 2011) est une imposante et remarquable biographie de Riad al-Solh, qui fut Premier ministre du Liban en 1943 lors de l'accession du pays à son indépendance. Il fut assassiné en juillet 1951 à Amman. Au-delà de la vie de cette personnalité politique de premier plan, c'est aussi tout un pan de l'histoire du Liban et du Proche-Orient qui est dévoilé dans cet ouvrage à partir de sources inédites (notamment arabes). En publiant la traduction de la conclusion de cet ouvrage, Transcontinentales inaugure une nouvelle rubrique Bonnes feuilles dont l'objectif est de signaler aux chercheurs et éditeurs francophones des ouvrages marquants de sciences sociales rédigés dans d'autres langues que le français et l'anglais. 
1 Riad al-Solh a été assassiné à l'âge de cinquante-sept ans. Au cours des dernières années de sa vie, il tint à rappeler son parcours et son expérience à ses jeunes collègues du Parlement, cherchant ainsi à aiguiser leur discernement. Son engagement précoce en politique (que l'on nommait jihad à cette époque), puis son départ, avaient occupé toute sa vie. Elles lui conféraient l'aura d'un vieux sage. Ces quarante années de carrière avaient conduit le jeune étudiant en droit d'un bureau d'Istanbul au poste de Premier ministre du Liban; il fut assassiné à Amman quelques mois après avoir quitté le pouvoir. Il fit face, au cours de son existence, à de nombreux défis. Il connut la prison, la condamnation à mort et l'exil. Il voyagea beaucoup et visita de nombreuses villes. Il côtoya les grands de ce monde et fréquenta les gros bras (qabadây) de Beyrouth et de Saïda. Il fut journaliste et homme politique, et passa d'innombrables heures dans les rédactions à lire, écouter et discuter. Les journalistes formaient son cercle le plus proche. Aucun autre politicien libanais ne bénéficiait d'un soutien aussi fort de la part de la presse libanaise, arabe et même internationale. Il fut aussi avocat. Le cabinet qu'il dirigea pendant quelques années était presque exclusivement fréquenté par des hommes politiques. Les gens se tournaient vers lui et il ne les évita ni durant son mandat ni à aucun autre moment de sa carrière. Il considérait toujours ceux qui le sollicitaient, durant son mandat ou à un autre moment. Lorsqu'il voulait se faire discret - par mesure de prudence - au cours de la période qui précéda son accession au pouvoir, il se retirait dans l'arrière-boutique d'un ami commerçant, Youssef al-Saddi. Il avait appelé ce lieu la "retraite de Sion », en référence à celui dans lequel le Christ et ses apôtres s'étaient réunis à Jérusalem avant qu'il ne fût crucifié.

2 Ce za'îm (dirigeant) du jihâd - le jihad de son temps, auquel les chrétiens n'hésitaient pas à se joindre - se présentait devant les gens avec un air soigné et empreint de grandeur. Son regard brillant, son sourire chaleureux et son caractère jovial transparaissent dans des centaines de photos. Sa calvitie ne lui avait laissé des cheveux que sur les tempes et l'occiput. Quelques mèches rebelles apparaissaient sous un tarbouche qui tanguait sur le côté droit de sa tête ronde, sa taille moyenne tendait elle aussi à se remplir avec l'âge et se distinguait par le dynamisme de ses mouvements... Il tenait parfois un chapelet entre les doigts et avait toujours une cigarette à la main. Il était mordu de blagues, prompt à en rire et à en raconter. Il avait un goût pour les histoires et pour la grande poésie, plus spécifiquement celle d'Imru'l-Qays, de Mutanabbi et de Ahmad Chawqi, qu'il fréquenta. Tout cela donnait à ses audiences une familiarité emplie de respect. L'homme était connu pour son habileté politique et pour l'étendue de son réseau d'influence. Ses réponses étaient toujours pertinentes, il était habile dans la discussion et la polémique. Il acquit ainsi la réputation d'avoir une grande force de conviction et de parvenir, dans les négociations qu'il entreprenait, au meilleur arrangement possible. Au cours de ses prises de parole en public, il était aidé par une voix qui portait, un sens du verbe, une précision dans le propos et une aptitude à formuler des idées simples et précises.

3 Riah al-Solh avait hérité de son père puis de sa tante, d'une vaste fortune foncière distribuée entre Beyrouth, Tripoli, Saïda et le Sud-Liban (et l'on dit même à Lattaquié). Il en dilapida l'essentiel durant ses voyages et ses exils, au cours de ses combats politiques ou pour venir en aide à des amis en difficulté. Quant au reste (ce qu'il possédait au Sud-Liban et qui n'était pas négligeable), il fut hypothéqué de 1921 à 1942. Riad al-Solh vécut donc endetté tout au long des décennies 1930 et 1940. On l'expulsa, au début des années 1930, de sa première résidence beyrouthine dans le quartier de 
Nâsra en raison de loyers impayés. Un ami de la famille Moussalim de Zahlé l'accueillit et l'hébergea dans un appartement de la rue Omar b. Khattâb dans le quartier de Ras alNab'. Riad al-Solh y demeura jusqu'à sa mort. Il ne put jamais réaménager l'appartement tant il ployait sous le poids de dettes placées sous la responsabilité du mufti de la République et dont la liste fut publiée après son assassinat.Le roi Abdel Azîz al-Saoud bâtit pour lui une maison qu'il aurait voulu lui-même construire dans des proportions plus petites, sans y parvenir. Ce quartier, qui s'étend des environs de l'aéroport à Bir Hassan, était convoité par de nombreux politiciens qui quittaient Ras al-Nab', Mazra'a et Basta al Fawqa pour s'y installer. Il s'agit des anciennes zones périphériques de Beyrouth progressivement intégrées au tissus social de la ville. Riad al-Solh faisait partie de ces nouveaux immigrés potentiels. Il s'endettait parfois pour aider les autres. Si l'on parlait beaucoup de corruption à l'époque de son gouvernement, presque personne n'osa l'accuser directement. Et, lorsque cela arriva, certains (comme Antoun Saadé) lui attribuèrent la propriété d'une rue dans une ville égyptienne que personne ne parvint jamais à trouver. Ses filles, Alia et Lamia, dans un article qu'elles publièrent peu avant son assassinat, révélèrent que l'équilibre financier de la maison reposait pour une grande part sur les épaules de leur mère et non de leur père.

5 Heureusement pour sa carrière politique, Riad al-Solh ne se conduisait pas en saint. Polémiste redoutable, il faisait face à ses adversaires, leur jouait des tours, leur dressait des embûches et savait déjouer celles qu'ils lui tendaient... Sa réputation dans le milieu fut installée lorsque l'on commença à parler de «la ruse de Riad al-Solh » et de ses "tours ». Le témoignage de Youssef Salem illustre parfaitement la relation qu'il entretenait avec la politique: "doué d'une pensée fulgurante, il considère que tout problème comporte une solution et toute impasse une issue ». Concentré sur son travail, Riad al-Solh fermait les yeux sur un certain nombre de choses qui auraient mérité d'être condamnées, préférant - selon ses propres termes - s'attacher à l'essentiel. Il défendait parfois l'indéfendable et assumait toutes les décisions des instances dirigeantes, même quand il ne les approuvait pas, au motif que lui-même gouvernait. Il n'hésitait pas non plus à réprimer les mouvements contestataires qu'il estimait menaçants pour la stabilité et la sécurité générales du régime en réclamant des droits de façon prématurée ou en se cachant derrière des revendications légitimes afin de déstabiliser le pays. On lui reprocha - à raison d'un point de vue juridique - d'avoir deux poids et deux mesures. Il évita ainsi d'alimenter, contre le régime, une contestation confessionnelle qu'il considérait, comme de nombreux autres dirigeants, comme plus dangereuse que n'importe quelle autre forme de contestation sociale. C'est la raison pour laquelle il adopta une position ambiguë dans ce genre de situation, prenant en compte les pressions qui s'exerçaient sur lui comme sur les autres politiciens, évitant au fossé de s'élargir entre les confessions.

6 Riad al-Solh fut au pouvoir à un moment où le pays faisait face à des dangers exceptionnels. Après la longue confrontation avec les Français durant le mandat et leurs ambitions incessantes, il y eut la guerre en Palestine et la menace de sa disparition, les troubles en Syrie et leurs implications explosives au Liban, les crises grandissantes dans les relations internationales au lendemain de la Seconde Guerre mondiale et les répercussions que tout cela pouvait avoir à l'intérieur du pays. Il considérait que ce cortège de menaces justifiait, de temps à autre, le recours à la 
fermeté, faisant passer l'entente dans le pays avant toute autre considération. Il estimait, en effet, que celle-ci constituait un bouclier pour le pays devant les dangers intérieurs et extérieurs qui planaient sur lui. Bechara el-Khoury reprocha à Riad alSolh, au tout début de leur mandat, de ne pas consacrer suffisamment de temps aux dossiers dont il avait la charge. Nous ne disposons d'aucune preuve susceptible d'infirmer ce témoignage. Mais l'homme ne se reposait ni ne dormait avant la fin de son travail. Saadi Mounla, l'un de ses héritiers et successeurs au poste de Premier ministre, ne cacha pas son admiration devant sa grande capacité de travail : "Je n'arrive pas à comprendre comment Riad bey al-Solh partage son temps entre sa vie et son travail ? C'est vraiment quelque chose d'étonnant. »

7 Sous prétexte de préserver l'unité du gouvernement, Riad al-Solh ferma les yeux sur de nombreux cas de corruption dans son entourage. On lui attribue d'ailleurs des propos (dont nous avons parlé et qui manifestent beaucoup d'atermoiement) soutenant que la corruption avait pour bénéfice d'orienter les corrompus vers le « profit » au lieu de les voir se jeter dans les bras de "l'impérialisme »! Aussi bien dans sa vie personnelle que dans l'exercice politique, en dehors de certaines occasions, Riad al-Solh rejetait le confessionnalisme. Il l'affronta même en faisant tout pour favoriser le rapprochement et l'égalité entre les confessions et en gouvernant de façon consensuelle. Tout cela dans une société qui avait hérité d'un lourd passif de discordes communautaires par rapport aux grandes décisions politiques. La société avait aussi hérité de disparités très profondes dans le développement social et urbain des régions, elles-mêmes marquées du sceau de la dissension confessionnelle, ainsi que dans la reconnaissance de leurs rôles historique et politique respectifs. Riad al-Solh continua malgré tout à chercher l'occasion politique qui lui aurait permis d'ouvrir une brèche dans le mur de la règle confessionnelle dominant l'ordre politique et modelant la société dans ses structures, ses équilibres et ses héritages historiques. Les disparités sociales provoquées par le confessionnalisme n'avaient pas échappé à Solh. La corruption qui les accompagnait non plus, tout comme les portes ouvertes au tout-venant qui permettaient l'ingérence des puissances étrangères dans le destin du pays, profitant des rivalités politicoconfessionnelles. Riad al-Solh sentait le danger que tout cela faisait peser sur l'équilibre du pays. Il était donc très sérieux lorsque, dans la déclaration ministérielle du premier gouvernement qu'il forma, il proclama son intention d'abolir le confessionnalisme. Il était tout à fait conscient qu'un objectif de cette nature ne pouvait se réaliser autrement que par la politique. Il fallait pour cela réunir un grand nombre de forces en présence afin de garantir et de protéger ce programme. Et, comme Solh était convaincu, il profita des quelques occasions d'unité qui se présentèrent à l'époque où il gouvernait pour promouvoir cet idéal et entreprendre de l'atteindre, en restant attentif à ce qui pouvait consolider cette position ou la compromettre. À maintes reprises, toutefois, il dut reculer et abandonner ce projet pour composer avec ses adversaires.

Riad al-Solh entretenait des relations cordiales avec certains de ses opposants. Elles dépassaient l'adversité politique et frisaient parfois la fascination. En témoignent les relations qu'il eut avec certains piliers du Bloc national libanais, Émile Eddé, son fondateur, et Assaad Akl, rédacteur en chef d'al-Bayraq, pendant les deux décennies au cours desquelles il s'opposa au mandat français et à ses agents et s'empara des rênes du pouvoir avec Bechara al-Khoury. Il prit le premier sous sa protection, au moment le plus délicat de sa carrière politique, quand il fut accusé de trahison. Riad al-Solh sortait alors d'une grande bataille contre les autorités mandataires qui avaient trouvé en Eddé leur meilleur partisan. Al-Bayraq était un des journaux les plus favorables à Solh. Il 
suivait de près l'actualité de son gouvernement et exprima ses regrets au moment de son décès. L'audace politique de Riad al-Solh s'inscrivait dans le prolongement de cette ligne de conduite. En modéré il avait abandonné tout attachement à un principe d'authenticité et refusait toute forme de blâme émanant des tenants de la pureté patriotique, surtout quand il s'employait à entraîner un adversaire dans une alliance circonstancielle pour en affronter un autre au cours d'un combat décisif. Il s'était tenu à distance égale de tous les blocs arabes et s'imposait comme un intermédiaire entre eux. Son audace se manifesta, entre autres, à l'occasion d'au moins deux initiatives qu'il conduisit, au cours des années 1920 et 1930, afin de convaincre le commandement sioniste de se rapprocher du bloc syrien dans sa lutte contre l'autorité mandataire. Sa capacité à combiner un positionnement radical et une certaine flexibilité constituait une autre manifestation de son cran. Elle lui permit de se tenir à égale distance des Hachémites qui s'opposaient aux Égyptiens et aux Saoudiens au moment de la guerre en Palestine.

Le mandat de Bechara al-Khoury et de Riad al-Solh fut aussi consacré à la mise en place des institutions et à l'initiation des grands travaux accompagnant la naissance d'un État indépendant. Ce mandat constitua, en un certain sens, l'édification de l'État lui-même. La mémoire collective n'a pas conservé les traces de l'âpreté des luttes politiques ayant marqué cette époque. La première d'entre elles, le combat pour l'indépendance, donna son nom à cette période et lui imprima sa marque au point de donner l'impression que rien d'autre ne s'y était produit. Cette époque, qui s'étend jusqu'en 1952, porte l'empreinte des affaires qui la façonnèrent en profondeur : la corruption, l'iniquité et l'atteinte à l'autorité de la loi par les familles politiques. La "révolution blanche » de 1952 avait pris la tournure d'un coup d'État militaire sur au moins un point : la volonté d'effacer ce que le précédent mandat avait réalisé sur le plan des institutions de l'État, de renouveler la structure sociale et de donner naissance à un bonheur qui ne cesserait de croître. Des témoignages de cette époque nous sont livrés dans un livre bien documenté savamment élaboré, préfacé par le président de la République et édité sous le titre Le Liban à l'époque de l'indépendance, publié en août 1947 à l'occasion de la première conférence culturelle arabe de Beit Mery. Nous en retrouvons dans un autre livre, critique et analytique, qu'est la somme en langue française réalisée par Gabriel Menassa, président de l'association libanaise d'économie politique, son adjoint, Joseph Najjar et un groupe de spécialistes. Cet ouvrage intitulé Plan de reconstruction de l'économie libanaise et de la réforme de l'État s'est appuyé sur une masse de données concernant l'État et la société libanaise depuis l'indépendance (voire avant lorsque cela s'est avéré nécessaire) jusqu'à la date de sa publication, en 1948. Sa parution constitua un événement et elle se déroula, malgré le ton parfois très critique des contributeurs, sous le patronage des plus hautes autorités de l'État, à commencer par Bechara alKhoury et Riad al-Solh.

11 Ces deux ouvrages, ainsi que d'autres sources qui les complètent, nous apprennent tant de choses sur l'urbanisme et l'édification des infrastructures de l'État indépendant que nous devons nous restreindre à l'essentiel.

Le mérite d'avoir mis en place le ministère des Affaires étrangères et le corps diplomatique revient à Sélim Takla, ministre des Affaires étrangères du premier gouvernement d'indépendance, et Riad al-Solh. Hamid Frangié en avait jeté les fondations à la veille de l'indépendance. Riad al-Solh et le commandant de l'armée

Transcontinentales, $10 / 11$ | 2011 
Fouad Chehab, qui étaient très proches, transformèrent " les unités spéciales » héritées du mandat en une armée libanaise, qui demeurait certes faible et peu équipée, mais qui s'était professionnalisée et avait réussi à se mettre à l'abri des ingérences politiques. L'organisation des autorités judiciaires et de leurs différents organes s'était poursuivie sous le mandat de Riad al-Solh par la suppression des tribunaux mixtes et leur accession au statut de troisième pouvoir dans l'État indépendant. La relation du judiciaire et du politique demeura toutefois une question en suspens. À l'époque de Bechara al-Khoury et Riad al-Solh, la monnaie nationale s'autonomisa, le système des impôts se renouvela et s'épura de ses archaïsmes. On refonda l'organisation financière de l'État et l'on mit en place la Cour des comptes. On élabora un Code du travail (1946, avec le gouvernement de Saadi Mounla), répondant ainsi à une revendication pressante des syndicats. On constitua aussi, au sein du ministère de l'Économie, un département des affaires sociales, embryon du ministère qui portera plus tard le même nom.

Il va sans dire que cette entreprise fut entachée de défauts. Certains provenaient du manque de savoir-faire, de moyens et de compétences. Les plus importants venaient de l'intrusion des politiques et des notables confessionnels. Riad al-Solh n'affronta pas ces problèmes avec la fermeté nécessaire. Il accorda la priorité aux aspects politiques qui l'emportèrent le plus souvent. Il n'en demeure pas moins que la force de son emprise sur le pouvoir et son indifférence aux profits personnels constituaient des garde-fous et permettaient, de temps en temps, d'enrayer les abus. Sa carrière politique est émaillée ainsi de nombreuses confrontations avec des entrepreneurs avides de pouvoir et d'influence. Quoi qu'il en soit, les fondements de l'édification de l'État et de ses institutions furent jetés à ce moment-là; la consolidation, la réforme et l'accomplissement reposaient désormais sur les épaules des générations futures.

Sur le plan de l'urbanisme et de la construction, Bechara al-Khoury et Riad al-Solh édifièrent des centaines d'écoles publiques dans les villes et les villages du Liban. Ils mirent sur pied l'enseignement secondaire, renforcèrent le syndicat des enseignants et fondèrent l'Université libanaise. Le livre de Gabriel Menassa, qui est la contribution la plus récente au sujet de l'enseignement public à cette époque, signale l'existence, en 1942-1943, de 248 écoles. Ce chiffre monte à 623 pour les années 1946-1947. Le nombre d'enseignants passe, pour la même période, de 402 à 1502 puis à 1802 en 1948 . Le nombre d'élèves évolue, quant à lui, de 22844 à 52 422. Le livre souligne la création de classes complémentaires dans onze de ces établissements, ainsi que d'écoles techniques. Il parle aussi d'un emprunt visant à construire la première école secondaire publique dans le pays. Il montre que le budget du ministère de l'Enseignement croît de manière notable en passant de 4,80 millions de livres libanaises en 1947 à 7,25 millions en 1948. La plupart de ces nouvelles écoles, dont nous évaluons le nombre à 600 pour l'année 1952, étaient petites. On louait, dans un premier temps, des salles partout où cela était possible. Elles permirent toutefois l'accès à l'enseignement à des dizaines de milliers de familles qui en étaient restées privées jusqu'alors, leur offrant une chance de changer de situation. Parallèlement, on publia en 1946 (sous le gouvernement de Samy Solh) le nouveau programme d'enseignement unifié. Il s'inspirait, dans ses orientations, des grandes lignes du programme d'enseignement national édicté dans la déclaration ministérielle du premier gouvernement d'indépendance. Il préservait les " acquis positifs » de la période mandataire, mais empruntait, dans la forme comme dans le contenu, une voie considérée comme nouvelle à cette époque, en accord avec l'esprit d'unité qui avait conduit le pays à son indépendance. 

et des avocats au cours des dernières semaines de Riad al-Solh à la tête du gouvernement. Ce dernier déclara au Parlement, au moment où il quittait le pouvoir : "Ce moment où je me tiens devant vous [...] pour vous demander, chers amis, de donner votre accord à la création de l'Université libanaise, est l'un des meilleurs de toute ma carrière parlementaire. On nous a demandé, par la voix de la jeunesse enthousiaste, de créer cette institution et nous la leur avons promis. L'Université verra bientôt le jour et elle rapprochera tous les enfants du pays. Elle ne constitue pas seulement un lieu d'enseignement [...]. Nous voulons qu'elle soit une université nationale. Répondez à l'appel de la jeunesse. » décennies qui suivirent, le visage de la société. Le profil des élites changea du point de vue de leur répartition régionale et confessionnelle. De nouvelles compétences virent le jour et se développèrent dans les villes comme dans les villages. Ce changement comporta, bien évidemment, des failles considérables. Comme il ne s'accompagna pas d'une politique adéquate, il contribua à propulser le pays dans des crises aux multiples causes et aspects. Il ne faut pourtant pas nier les mérites de cette réforme qui comblait un besoin et qui créa les bases d'une allégeance à l'État et d'un sentiment d'appartenance national. Ces accomplissements ne connurent pas la même fortune dans les périodes qui suivirent, et ne résistèrent pas aux orages politiques qui frappèrent le pays. Mais, leur mise en place, au lendemain de l'indépendance, répondait à un besoin et à un rêve partagés.

Beyrouth vit le jour au cours des derniers jours de Riad al-Solh au pouvoir. On édifia aussi à cette même époque le palais de l'Unesco et ses annexes, afin d'accueillir le troisième congrès de l'Organisation à l'automne 1948. Hamid Frangié, ministre des Affaires étrangères dans le gouvernement de Riad al-Solh, supervisa le chantier, les préparatifs et le déroulement du congrès avec une célérité et une efficacité saluées. Nous pouvons compter, parmi les innovations positives du mandat de Bechara al-Khoury et Riad al-Solh, la fondation de ministères et de nouvelles directions publiques ainsi que la modernisation d'un grand nombre d'institutions. On créa de nouveaux bâtiments et on loua des palais et des édifices pour les accueillir. On construisit des tribunaux, des bâtiments publics et techniques, des hôpitaux et des commissariats que l'on répartit selon les régions (muhafazat) et les municipalités (baladiyyât)... Riad al-solh dit qu'on ouvrit aussi des prisons.

18 L'ère Bechara al-Khoury/Riad al-Solh se caractérisa par une réforme urbaine de la capitale, avec les projets Egli et Ecochard. Cette initiative servit de modèle à bien d'autres qui la suivirent et qui connurent, à son instar, des fortunes diverses. À la même époque, le réseau routier fut développé entre les régions libanaises et les accès aux villes furent élargis et modernisés. L'eau courante et l'électricité se généralisèrent et atteignirent des régions qui n'y avaient pas encore eu accès. Le chemin qui mènerait à l'abolition de la misère restait cependant très long. On installa de même le réseau téléphonique automatique à Beyrouth et l'on construisit le grand édifice des bureaux centraux de la poste sur la rue qui porte aujourd'hui le nom de Riad al-Solh.

19 Parmi les grands travaux de l'époque, le nouvel aéroport international de Beyrouth à Khaldé remplaça progressivement celui de Bir Hassan, trop petit et trop proche de la ville. Des avenues rectilignes furent percées pour relier l'aéroport à la ville, à commencer par l'avenue Bechara al-Khoury elle-même. Des grands boulevards furent 
tracés par la suite, traversant le bois des pins et constituant les nouvelles aires de développement de la ville. La route menant à l'aéroport devint, pendant le quart de siècle suivant, un espace de verdure, de repos et de promenade pour les habitants de la ville. La loi de 1950 avait fixé les règles d'urbanisme de la zone, ce qui en préserva le caractère et l'élégance jusqu'au déclenchement de la guerre en 1975. L'aéroport occupa, tout au long de cette période, une place importante au cœur des communications entre l'Orient arabe et le reste du monde, de même qu'il s'imposa comme une artère centrale de l'économie du pays. Ajoutons que les règlements des ports de Saïda et de Beyrouth furent établis au même moment, que le terminal pétrolier de Tripoli, qui recevait du pétrole brut de Kirkouk, fut élargi, et que l'on construisit, aux environs de Tripoli, une nouvelle centrale de raffinage, plus importante que l'ancienne. Saïda devenait, de son côté, le terminal du pipeline transarabique et le point de départ de son exportation maritime. On y construisit aussi une station d'assainissement. Pour aller au-delà de la polémique légitime sur l'écart entre les changements réels que ces infrastructures apportèrent et les attentes qu'elles avaient engendrées, soulignons qu'elles avaient procuré un emploi à des milliers de Libanais et constitué, par le biais des impôts et des taxes, l'une des sources de revenus les plus importantes de l'État.

À Saïda et dans le Sud, le mandat n'avait laissé aucune construction notable. Riad alSolh, qui était le député de ces régions, initia, avec Ahmad al-Assad, son ministre des Travaux publics, le célèbre projet de Qasimiyya, avec ses proportions monumentales et ses bénéfices considérables. Il devait irriguer toute la plaine qui s'étend de Saïda à Tyr. L'annexe de Ras al-Ain, au sud de Tyr, venait compléter ce dispositif. L'ingénieur hydraulique Ibrahim Abdel Al devint le directeur général des travaux publics et gagna les dirigeants politiques à ces deux projets d'irrigation, comme à bien d'autres partout dans le pays. Malgré des niveaux de réussite très variés, la quantité de travaux réalisés en si peu de temps et avec si peu de moyens étonne: projets d'irrigation et autres installations hydrauliques, centrales électriques, etc. Car les infrastructures de Qasmiyya et Ras al-Ain ne sont que des éléments d'une série de projets visant à exploiter les eaux des Nahr al-Kébir et Nahr al-Bared au Akkar, du Nahr al-Jawz à Batroun, des sources de Yammouneh dans l'arrière-pays de Baalbek, du Litani et du Laboué dans la Békaa. On devait profiter du Nahr al-Assal pour l'eau potable, de Nab' alRay dans le Kesrouan pour l'irrigation. On voulait amener l'eau du Barouk dans le caza' d'Aley et créer une centrale électrique sur le Nahr Ibrahim.

21 À Saïda, l'hôpital et la première école secondaire publiques du Sud furent inaugurés sous le patronage de Riad al-Solh. Il posa la première pierre du nouvel édifice de la municipalité et édifia le stade municipal et la cité du travail. Il construisit le grand hôtel de Saidoun, que la municipalité s'engagea à faire exploiter par la chaîne d'hôtels Tanios. Il perça la rue principale de la ville qui porte aujourd'hui son nom. Elle devint rapidement le centre du commerce et des affaires et permis à la ville de s'étendre progressivement vers l'Est. La plupart de ces services faisaient partie d'un réseau d'infrastructures plus large qui couvrait tout le territoire et que l'on trouvait dans la plupart des capitales régionales du pays.

On restaura aussi le palais de l'émir Béchir Chehab II à Beit al-Dîn qui était tombé en ruine. On rapatria même les restes de l'émir qui avait été enterré dans une église arménienne catholique d'Istanbul afin qu'il repose en son palais. Leur retour au pays se déroula à l'été 1948 et fut célébré de manière très officielle. Sabri Hamadé et Riad alSolh prononcèrent des discours à Beyrouth et Bechara al-Khoury à Beit al-Dîn. On 
transforma l'une des ailes du palais en un musée de la période féodale libanaise. L'autre devint le siège estival de la présidence de la République. Le jardin retrouvait quant à lui ses splendeurs d'antan. Le président de la République veilla personnellement au développement du projet et tira une grande satisfaction de ce qui avait été accompli.

[...]

Il faudrait, pour compléter ce tableau et prendre la mesure de l'héritage laissé par Bechara al-Khoury et Riad al-Solh, parler de la dimension législative de leur legs. La première chose qui vient à l'esprit, sans aucun doute, est le célèbre amendement de la Constitution au moment de l'indépendance, en novembre 1943, et tous les textes qui se succédèrent et qui permirent d'élaborer les infrastructures juridiques et exécutives de l'État indépendant. Nous avons signalé, en différents endroits de ce livre, celles que nous considérons comme les plus significatives. Si nous laissons de côté la période de l'indépendance, il nous faut considérer les deux législatures parlementaires qui accompagnèrent les gouvernements que présida Riad al-Solh. Bien que quantitativement peu importantes, les nouvelles lois s'attachent à des domaines très divers et se caractérisent par des perspectives fondamentales. Nous devons y ajouter le nombre important de décrets qui accompagnèrent leur vote au Parlement.

Concernant les lois qui régissent le système économique et financier du pays, la loi du commerce maritime, promulguée le 18 février 1948, complète la loi du commerce terrestre édictée par décret-loi du 24 novembre 1942, c'est-à-dire avant l'indépendance. De nombreux décrets s'y ajoutent. Promulgués pour l'essentiel entre 1948 et 1950, ils devaient faciliter les transactions postales et l'organisation des relais télégraphiques. Ils débouchèrent sur la signature de contrats et de protocoles internationaux, dont ceux qui furent adoptés au cours de la conférence sur les communications avec et sans fil d'Atlantic City, et aux conférences internationales sur l'organisation postale de Paris de 1947 et 1949. Le 11 janvier 1949, c'est au tour de la loi de réglementation aérienne d'être édictée. Elle diffère des deux précédentes en raison de ses aspects techniques. Elle les complète cependant sur un grand nombre de points. Signalons aussi l'ordonnance du 8 avril 1950 qui reconsidère «l'organisation des taxes douanières sur les produits destinés à la réexportation ». L'objectif était d'encourager l'activité de transit en raison de sa place prépondérante dans l'économie libanaise.

Toujours sur le même plan, la loi du 22 avril 1948 autorise l'adhésion de l'État au Fonds monétaire international et à la Banque mondiale qui sont, comme chacun sait, deux instruments de la gouvernance financière mondiale au service des pays riches. Le 24 mai 1949 (c'est-à-dire un an avant la rupture monétaire avec la Syrie), la loi monétaire fut promulguée et suivie, trois jours plus tard, du décret relatif à la protection monétaire, préservant la valeur de la livre libanaise pendant une longue période. Les lois de 1956 sur le secret bancaire et sur la monnaie et le crédit de 1963, sous Camille Chamoun et Fouad Chehab, les amendèrent par la suite.

L'attention portée aux aspects commerciaux et financiers de l'économie nationale fut complétée par une politique touristique. La loi régissant les activités des organisations et des individus travaillant dans le secteur du voyage est promulguée en juin 1949. Elle fut précédée, le 14 janvier de la même année, par la loi régissant les investissements dans les secteurs de l'hôtellerie, des centres de loisirs, de la restauration, des cafés et des bars. L'État encadra ce secteur en créant, par la loi du 7 mai 1948, un «Commissariat général du tourisme, de l'estivage et de l'hivernage ». 
Sur un autre plan (mais qui relève aussi de l'économie), les accords régissant les modalités de passage des hydrocarbures sur le territoire de la République libanaise furent conclus le 3 mars 1947 avec la société Tap-Line. La loi du 29 juillet 1950 entérine l'accord entre le gouvernement libanais et la Mediterranean Refining (Medrico). Nous avons beaucoup parlé, dans notre livre, des conflits qui ont accompagné la signature de ces accords et des amendements qui s'ensuivirent.

Parmi les lois essentielles du mandat Bechara al-Khoury/Riad al-Solh figure celle qui encadre les professions libérales. Seul le métier d'avocat bénéficiait, de longue date, d'une réglementation qui en fixait les conditions d'exercice et qui établissait son organisation syndicale. Le 26 décembre 1946 (c'est-à-dire juste après le retour de Riad al-Solh à la tête du gouvernement), la loi sur l'exercice de la médecine au Liban fut promulguée et le dernier jour de l'année, celle qui enjoignait la création de deux syndicats de médecins. Suivit le 17 juillet 1947, une loi qui fixait à deux sessions l'examen du Colloquium. La loi du 29 novembre 1947 encadra l'exercice de la psychiatrie, et fut suivie des lois du 27 juin 1949 instaurant l'Ordre des psychiatres. La loi réglementant la médecine vétérinaire vit le jour le 10 février 1948, et le 10 janvier 1951 (au cours du dernier mois de Riad al-Solh à la tête du gouvernement) la loi concernant l'exercice du métier d'ingénieur ainsi que la mise en place de deux Ordres des ingénieurs de Beyrouth et de Tripoli.

[...]

Si les bouleversements qui affectèrent le pays les années suivantes ont effacé et détruit presque toutes traces de ces efforts fondateurs, il reste de Riad al-Solh un projet d'apparence plus modeste, son programme politique. Il reste aussi son style délicat, sa patience et son obstination à vouloir réaliser ses projets. Cet homme s'entoura, lorsqu'il était au pouvoir, de représentants de toutes les tendances politiques qui traversaient le pays. Il fallait beaucoup de patience et de sagesse pour concilier l'entreprise de construction nationale et le maintien de l'unité nécessaire à la garantie de ce travail. Notre homme était conscient du rôle primordial de la politique dans la gestion des affaires humaines et la réforme de l'État. Il considérait qu'elle devait primer sur toute autre considération : la lutte contre les agressions, la réforme de l'État, l'urbanisme et la construction. Seul le travail politique, quand il s'accompagne de moyens, de plans et de l'adhésion de l'ensemble des acteurs, révèle le potentiel de toutes les autres activités, la justesse de tout travail et le degré de bien et de mal qu'il contient. Il vérifie surtout, par l'épreuve du réel, la correspondance entre le principe qui dirige l'action et sa réalisation, en montrant s'il a conduit ceux qui ont pensé y trouver le chemin du bonheur éternel à la catastrophe ou non. Riad al-Solh militait, dans sa jeunesse, pour l'unité arabe. Il resta fidèle à ce principe toute sa vie. Il n'a cependant pas hésité à faire marche arrière quand il a vu que cela pouvait provoquer la division et la destruction de la nation. Il était indépendantiste et a trouvé que ce principe pouvait rassembler les rangs. Il alla le plus loin possible dans cette direction, mais il mesurait ses pas et considérait toutes les possibilités avant d'entreprendre quoi que ce soit. Il était courageux et acceptait toutes sortes de sacrifice mais faisait preuve d'une grande humilité. Il se renseignait sur les avantages de telle ou telle chose et gardait toujours les portes ouvertes au dialogue et à la discussion. Il seréservait un espace de manœuvre, ne se laissant pas gagner par l'entêtement aveugle et évitant bien de se faire prendre au charme de ses propres formules oratoires. 

indépendante du contexte et des équilibres politiques. Il s'agissait d'une réponse de grande sagesse aux défis politiques qui avaient germé au moment de l'accord et qui continuèrent pendant bien des années. Le pacte n'était pas seulement dirigé à l'intérieur, contre les tenants de l'unité arabe d'une part et les isolationnistes d'autre part. Il répondait aussi aux demandes françaises (et anglaises par la suite) d'établir des traités et des relations privilégiées avec le Liban et de mettre en place, ce qui finit par être connu dans les années 1940, une forme de "défense du Moyen-Orient ». Il offrait aussi une alternative au projet de la Grande Syrie et du croissant fertile puis aux atermoiements des putschistes syriens entre un projet d'unité syro-irakienne et un projet d'unité arabe complète, proposé par le gouvernement Qudsi à la fin du mandat de Riad al-Solh. Face à tous ces défis, le pacte national a tiré sa force de sa capacité à l'emporter sur tous les courants qui déchiraient la société libanaise et à attirer des responsables politiques populaires et expérimentés qui avaient commencé par s'y opposer. Riad al-Solh en faisait partie et il en avait pris la tête.

Riad al-Solh a trouvé dans ce Liban arrivé à l'indépendance la meilleure solution pour les Libanais et les Arabes. Lui qui s'impliqua dans l'établissement et la construction de cet État, accepta ce que cela impliquait d'assiduité et de constance, voire même d'opposition à des puissances proches et lointaines. Il ne remit jamais ce choix en question et refusa les blâmes de ceux qui en souhaitaient l'échec et voulaient voir en lui un porte-parole de leur volonté dans un pays dont il était devenu le garant. Riad al-Solh permit au modèle indépendantiste de l'emporter ailleurs dans les pays arabes dont il accompagna le cheminement. Il le considérait comme le meilleur, parmi toutes les espérances actives à l'époque, ce qui ne signifie pas qu'il fut aisé à accomplir. Il consentit au projet d'unité sous la forme de la Ligue arabe, non comme un préalable à la dissolution nécessaire des nations indépendantes, mais pour faire de ces dernières les garantes de la solidarité, de l'intégrité et de l'efficacité de l'alliance. En tant que politique authentique, Riad al-Solh détestait voir les militaires s'impliquer dans les affaires de la cité, avec leurs méthodes brutales et leur tendance à réduire le pluralisme par la force. Il n'assista pourtant à l'arrivée au pouvoir des militaires que dans un seul pays, qu'il connaissait bien. Ce n'était que le prélude à une généralisation du phénomène à de nombreuses autres contrées du monde arabe, avec des méthodes bien plus brutales et arbitraires que celles que Riad al-Solh avait observées ou affrontées. Il ne réussit pas dans tout ce qu'il avait entrepris. Cela n'était de toute façon pas possible, car ce genre de quête n'a pas de fin. L'évolution de l'Orient par la suite apparaît comme un renversement des aspirations, des alliances et des méthodes politiques de Riad alSolh. Des notions comme la liberté, l'indépendance des peuples, la solidarité dans le cadre du respect de la souveraineté des États, le respect du pluralisme dans les sociétés, avaient été évoquées par Riad al-Solh, ou étaient apparues par la suite pour rendre compte de son action. Elles restèrent à même de s'imposer comme des objectifs imprescriptibles, même si l'on semblait s'en éloigner. Il n'y a pas d'alternative à ces valeurs élémentaires qui constituent la quintessence d'une époque et d'une histoire, et les moyens politiques d'en prendre conscience et de le préserver restent complexes et sinueux. Voilà ce que Riad al-Solh nous a laissé de plus précieux.

Dans la déclaration ministérielle $d u$ troisième gouvernement qu'il forma (en décembre 1946), il a explicité ce que voulait dire la formule: "nous avons donné naissance à l'État, il nous reste à construire la nation ». Dans les décennies qui suivirent 
sa disparition, nous nous sommes employés à renverser cette proposition. La formule de Riad al-Solh ne doit pas nécessairement nous conduire à changer celle que nous avons adoptée après lui. Nous avons traversé une expérience sanglante et douloureuse qui détermine ce que nous disons. L'idéal de Riad al-Solh mérite cependant d'être pris en considération. Il s'y trouve peut-être une vérité plus importante et plus profonde que ce que nous nous imaginons. 